\title{
Modeling the Age-Dependent Infectiousness of Diseases: An Integral Equation Approach
}

\author{
Rathgama Guruge Uma Indeewari Meththananda ${ }^{1, \text {, }, ~ N a l e e n ~ C h a m i n d a ~ G a n e g o d a ~}{ }^{2}$, \\ Shyam Sanjeewa Nishantha Perera ${ }^{3}$ \\ ${ }^{1}$ Department of Spatial Sciences, General Sir John Kotelawala Defence University, Sooriyawewa, Sri Lanka \\ ${ }^{2}$ Department of Mathematics, University of Sri Jayewardenepura, Nugegoda, Sri Lanka \\ ${ }^{3}$ Research \& Development Centre for Mathematical Modeling, Department of Mathematics, University of Colombo, Colombo, Sri Lanka \\ Email address: \\ umameththananda@gmail.com(R. G. U. I. Meththananda),naleen@sjp.ac.lk(N. C. Ganegoda),ssnp@maths.cmb.ac.lk(S. S. N. Perera) \\ ${ }^{*}$ Corresponding author
}

\section{To cite this article:}

Rathgama Guruge Uma Indeewari Meththananda, Naleen Chaminda Ganegoda, Shyam Sanjeewa Nishantha Perera. Modeling the Age-Dependent Infectiousness of Diseases: An Integral Equation Approach. Mathematical Modelling and Applications.

Vol. 4, No. 1, 2019, pp. 10-14. doi: 10.11648/j.mma.20190401.12

Received: March 30, 2019; Accepted: May 14, 2019; Published: June 4, 2019

\begin{abstract}
Many mathematical models developed through differential equations to describe the age dependent infectiousness of diseases, face the complexity of modelling heterogenic behavior of transmission. There, many of the cases assume the host to stay in the same risk class regardless of the age of the hosts. The proposed model mimics the infectiousness according to the age-scale of an individual via integral equation approach. This model indicates the applicability of Fredholm type integral equations with degenerated kernel. Introducing biological, behavioral and environmental influences provokes to address the accumulating nature of different factors in modelling the risk of getting infected. The risk of getting infected is modeled by the inability of responding with acquired immunity and the accumulated risk given from the other individuals in each age group via the mobility patterns. Within this approach environmental stimulus are modeled via periodic functions in order to describe the stochastic behavior of the spreading capabilities. In this study, the behavioral analysis evaluates the maximum risk of getting infectious in the considered parsimonious approach. And the sensitivity analysis describes the contribution of the mobility risk and stochastic nature on the overall risk. Further the model guides to formulate hypotheses and data collection strategies to measure the risk of a disease.
\end{abstract}

Keywords: Age Dependent, Degenerated Kernel, Infectiousness, Integral Equations

\section{Introduction}

In an epidemiological and public health standpoint, riskstructured susceptible-infected-recovered (SIR) models provide many insights into the dynamics of infections. But it generally assumes the host to stay in the same risk class irrespective of their age. However, the formalism of agestructured models accommodates the dynamics of individual aging process. These age-structured models are useful in providing an insight into the future epidemic behavior and to determine the proper control measures. In assessing the spread and morbidity of infectious diseases, infectiousness of the infected host and the susceptibility of uninfected individuals are main factors. Here the infectiousness refers to the ability of infecting another susceptible by already infected person. In biological perspective it is critical to assess this ability but primarily the percentage of infected individuals in each age group can be considered as a fair indicator. The biological influences, behavioral influences and environmental influences are three major components that can influence the infectiousness [1]. The biological and behavioral influences differ with the age of individuals. The environmental influences may have different interpretations according to the context addressed, as it could describe all the external stimuli effect on the transmission of the disease.

Mathematical models can be used to link these influences to observe the dynamics of infection. This study focuses to mimic the infectiousness according to the age-scale. In public health perspective, the individuals in their late teenage to 
twenties tends to be more vulnerable in getting into infections due to day-to-day mobilization [2-4]. It is a key factor in behavioral influence. Therefore the contact patterns of susceptible with infected hosts and susceptible with disease carrying agents relates with the mobility patterns of individuals which depends on the age [1]. As a biological influence, the dynamics of the disease in the body depend on the features of an individual immune system is also relies on the age of individuals. Depending on the disease, the environmental influence may describe the impact of climatic variation, impact of vector breeding and stochastic behavior of many natural phenomenon. Environmental influences cater the dynamics of disease causing pathogens such as virus, bacteria, parasites not only in host but also in vectors. For an instance vector mosquito involve in diseases like Malaria, Filariasis and Dengue.

Many approaches to capture these heterogeneities in transmission has been proposed via systems of differential equations [5]. More advanced models are with partial differential equations catering both time and age as independent variables [6-8]. It remains a considerable challenge to determine the parameters to model disease transmission in different age groups. In addition, systems with integro-differential equations have also been developed [9]. However, the proposed integral equation approach differs from usual SIR models due to the sequential progression through age. It simply requires only one type of equation to incorporate acquired immunity and mobility along with some external stimuli behavior. It would easily illustrate general behavior of infectiousness occurring as an accumulated process.

\section{Model Formulation}

The model proposed here describes the risk of being infectious in different age groups. It is first observed that the risk is related with inability to respond with acquired immunity and the risk accumulated due to mobility of hosts. Thus, a model can be established by considering that the excessive risk is proportional to accumulated experience of the risk due to other individuals of each age group. Here in expression (1), $f(a)$ is considered as a function of age $a$ representing the inability of responding with acquired immunity and $u(a)$ represents the overall risk level. Then $u(a)-f(a)$ is the excessive risk at the age $a$. Here the accumulation is governed by $K(a, t)$ later called the kernel of integral equation. The integration is taken over all ages, thus it is considered the average life span $L$ as the upper limit.

$$
u(a)-f(a) \propto \int_{t=0}^{L} K(a, t) u(t) d t
$$

where $K(a, t)=\sum_{k=1}^{n} A_{k}(a) B_{k}(t)$

By introducing a proportional constant $\lambda>0$, model equation becomes;

$$
u(a)=f(a)+\lambda \int_{0}^{L} K(a, t) u(t) d t
$$

The proportional constant shows the incorporation of the accumulation given by the integral over the lifespan to the excessive risk $u(a)-f(a)$.

One composition of the kernel $K(a, t)$ can be proposed as $\sum_{k=1}^{n} A_{k}(a) B_{k}(t)$. This describe the infectiousness in terms of who infected who and how by age-dependent behavioral influences via $A_{k}$ 's and external stimuli via $B_{k}$ 's. As a parsimonious approach, the number of terms needed to describe the infectiousness is reduced to one factor in each category as in Eq. (3).

$$
u(a)=f(a)+\lambda \int_{0}^{L} A_{1}(a) B_{1}(t) u(t) d t
$$

The risk of infectiousness can be described by the above model in Eq. (3) where the overall risk is derived by risk due to the mobility of each individual weighted by the accumulated infectiousness of the other individuals. As, SIR models assume a constant rate of infectivity and a constant rate of recovery, here in this model $B_{1}(t) u(t)$ describes the infectiousness of the other individuals where the individuals who got certain risk of getting infected may show the certain capability of infecting others $[10$, 11]. Hence summing over the lifespan provides the total infectiousness of the population, as an individual may interact with individuals in all the age groups.

In the process of assessing the applicability of this model, several hypothetical curves for biological, behavioral and environmental influences have been introduced. As described earlier, $f(a)$ represents the inability to respond with the acquired immunity at a particular age $a$.

$$
f(a)=1-0.9 * \frac{e^{\rho(a-\alpha)}}{1+e^{\rho(a-\alpha)}}
$$

where $\alpha$ and $\rho$ are parameters which represent the boosting age and the boosting duration of general immunity level in the body. It is observable that the immunological response is low at lower ages due to the lack of experience with the diseases, but it may boost and attain a certain level with the maturity as a result of the exposure to different pathogens. The behavioral influence is described as the risk level due to mobility. In many host populations it is evident that the mobility carrying higher risk in middle ages and declined with aging process [2-4]. That nature of mobility is modeled by

$$
A_{1}(a)=\frac{a^{2} e^{\left(-\frac{a}{\beta}\right)}}{4 \mathrm{e}^{-2} \beta^{2}}
$$

where $\beta$ is a parameter that represents the peak age of mobility. The function

$$
B_{1}(t)=\sin (\mu \pi t) ; \mu \in \mathbb{Z}
$$

is merged to the model as a periodic function to allow the stochastic behavior due to environmental influences. In this approach $B_{1}(t)$ is utilized to vary the capability of spreading the infection. There is a possibility of choosing a suitable stochasticity by considering a central measure for the randomness described by different periodicities in $\sin (\mu \pi t)$. Thus, the model output $u(a)$ adequately describes the general dynamics of the age-dependency of infectiousness. 


\section{Model Solution}

As Eq. (2) falls under the classification of the Fredholm integral equations, it owes several attributes in solving according to the characteristics of kernel $K(a, t)$ [12]. The structure of $K(a, t)$ used in Eq. (2) is called degenerated kernel in which the solving technique is based on transforming the equation into a system of linear equations.

In the proposed model, as $K(a, t)=A_{1}(a) B_{1}(t)$, the solving technique converts it in to Eq. (7)

$$
u(a)=f(a)+\lambda A_{1}(a) c_{1}
$$

where $c_{1}=\int_{0}^{L} B_{1}(t) u(t) d t$

The solution to the original equation counts on $c_{1}$ value. It can be figured out by converting Eq. (7) into a linear equation given below.

$$
c_{1}=f_{1}+\lambda A_{11} c_{1}
$$

where $f_{1}=\int_{0}^{L} B_{1}(t) f(t) d t$ and $A_{11}=\int_{0}^{L} B_{1}(t) A_{1}(t) d t$.

Finally, the solution for $u(a)$ is obtained as follows.

$$
u(a)=f(a)+\lambda c_{1} A_{1}(a) \text { where } c_{1}=\frac{f_{1}}{1-\lambda A_{11}}
$$

Now the solution implies that the excessive risk is a weighted function of behavioral influence in which $\lambda \frac{f_{1}}{1-\lambda A_{11}}$ provides the weighing coefficient.

In the following illustration in Figure 1, $f(a)$ depicts a situation of high risk in younger ages and later saturated in lower risk with maturity. Setting the lifespan into 80 years and hypothetical curves for $f(a)$ and $A_{1}(a)$ within the range $[0,1]$ allows an easier base for comparison with $u(a)$.

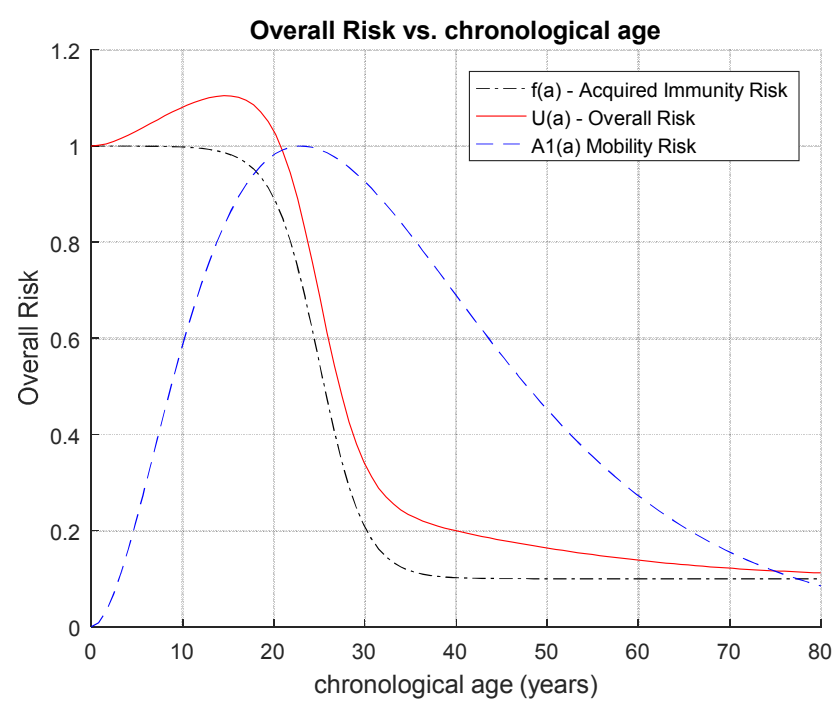

Figure 1. Age-dependent overall risk of infectiousness due to mobility, acquired immunity and environmental stimuli. | Black dashed line: inability of responding with acquired immunity when $\rho=0.4, \alpha=25$. $\mid$ Blue dashed: risk due to mobility when $\beta=11.5\left|B_{1}(t)=\sin (2 \pi t)\right|$ Red solid line: overall risk of infectiousness.

Figure 1 shows a general validity as overall risk level indicated by $u(a)$ is higher than $f(a)$, indicating the influence due to mobility. Moreover, overall $u(a)$ has approached a peak between 10 and 20 years and latter part settles with a saturation. These two attributes are not observable simultaneously in either $f(a)$ or $A_{1}(a)$. The two influential functions $f(a)$ or $A_{1}(a)$ carry either a peak (in $A_{1}(a)$ ) or a saturation (in $f(a)$ ), but not both phenomena.

It is evident from Eq. (9), the overall risk is subjected to be changed by varying weighing coefficient $c_{1}$. The following illustration in Figure 2, emphasizes that if positive $A_{11}$ occurs, then this overall risk no longer holds for every $\lambda$ value. Further, if negative $f_{1}$ together with negative $A_{11}$ appears, the overall risk can wane even with high mobility patterns. This shows unreliability in biological viewpoint.
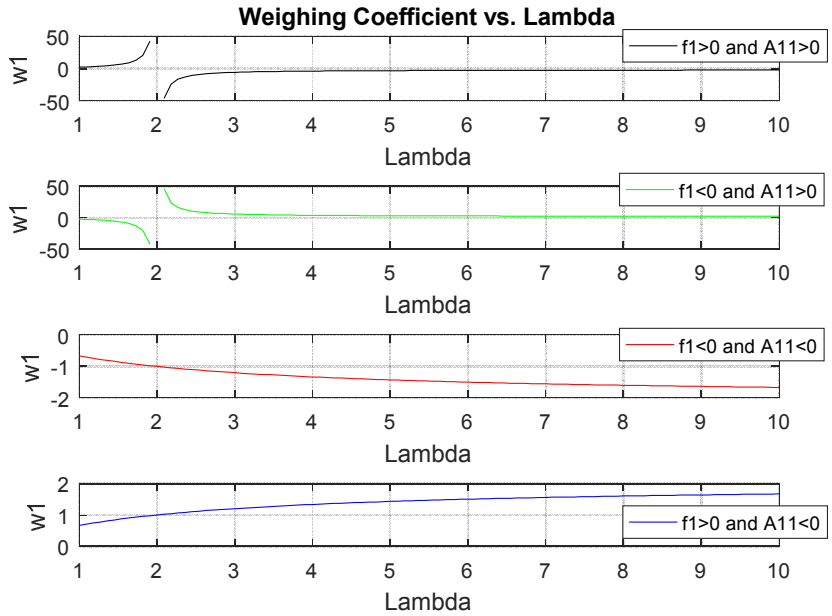

Figure 2. Contribution of weighing coefficient $w 1=\frac{\lambda f_{1}}{1-\lambda A_{11}}$ in different functional behaviors.

These types of different situations imply the importance of identifying proper and reliable influences for $f(a), A_{1}(a), B_{1}(t)$.

\section{Sensitivity Analysis}

As the model solution implies that the excessive risk is a weighted function of behavioral influence, it indicates effect of $\lambda$ and $\mu$ parameters over the sensitivity of $u(a)$.

Case 1: Sensitivity analysis for different $\lambda$.

This model acquaints that there is a possible maximum of overall risk which can be addressed with proper control measures such as vaccination, restricting mobility patterns etc. The simulation of overall risk with different proportional constant $\lambda$, reaches to a maximum for positive $f_{1}$ and negative $A_{11}$ as the contribution $(\lambda)$ increases. Hence the maximum overall risk or upper bound of the overall risk at different ages can be illustrated by

$$
\lim _{\lambda \rightarrow \infty} f(a)+\lambda \frac{f_{1}}{1-\lambda A_{11}} A_{1}(a)
$$




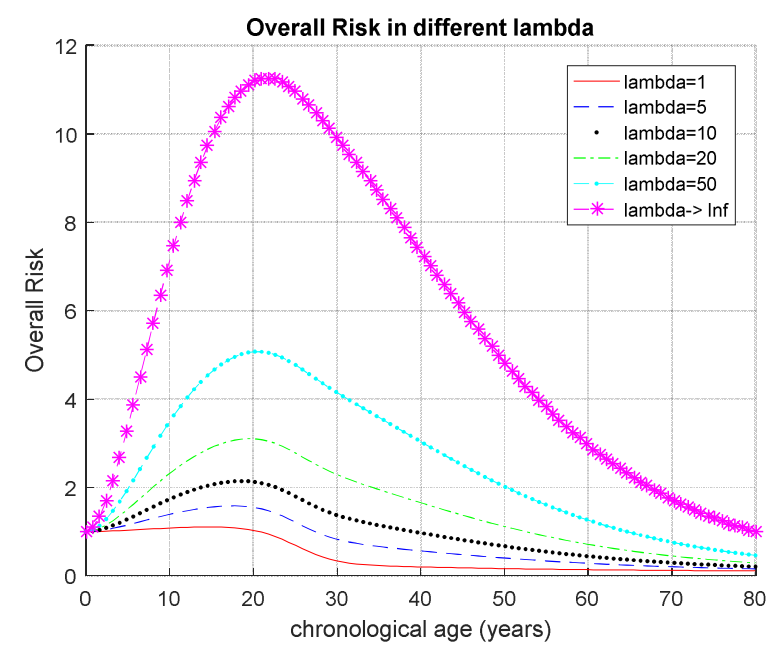

Figure 3. Sensitivity analysis on age-dependent risk of infectiousness inability of responding with acquired immunity when $\rho=0.4, \alpha=25$ and risk due to mobility when $\beta=11.5$ with $B_{1}(t)=\sin (2 \pi t)$

Figure 3 on sensitivity analysis of $u(a)$ with different $\lambda$, clearly visualizes the contribution of the accumulation of behavioral and environmental influences over the lifespan to the overall risk. What readily observable is that peaks slightly move towards higher ages and saturation may vanish with higher $\lambda$. The analysis on Figure 3 indirectly paves the way to estimate suitable $\lambda$ for a host population by comparing with another population. In that purpose, the risk level can be modeled via the prevalence of the disease by age. Such observational and experimental findings are available for some diseases in literature supporting this claim [13]. Further the excessive risk of proposed hypothetical curves may not drastically increase with $\lambda$, since the weighing coefficient $\lambda \frac{f_{1}}{1-\lambda A_{11}}$ in Eq. (10) is bounded as $\lambda$ increases. This illustrates that the possibility of identifying the required biological influence in order to not to get infected even with the maximum behavioral influence.

Case 2: Sensitivity Analysis for Different $\mu$ of Environmental Influence

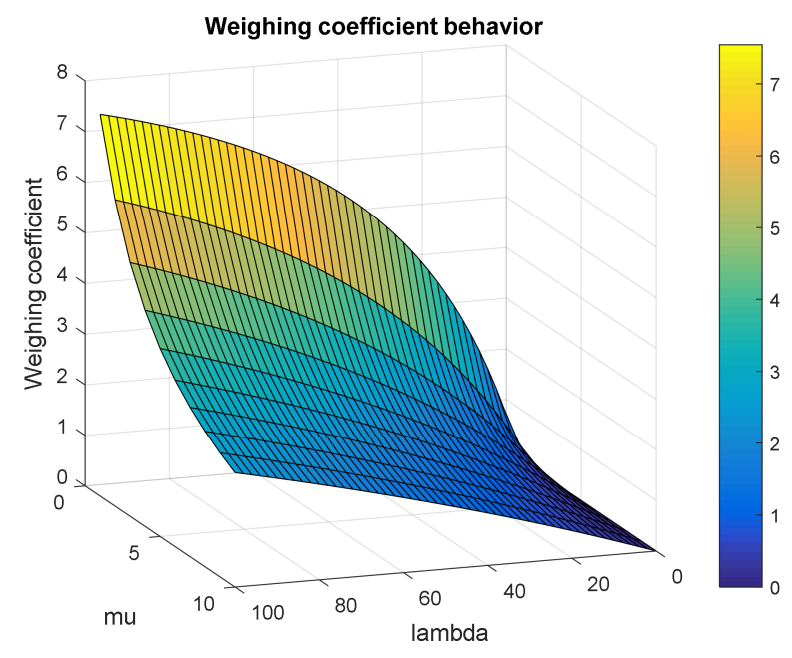

Figure 4. Weighing coefficient behavior $\left(\lambda \frac{f_{1}}{1-\lambda A_{11}}\right)$ at varying $\mu$ and $\lambda \mid f_{1}=$ $\int_{0}^{L} \sin (\mu \pi a) f(a) d a$ and $A_{11}=\int_{0}^{L} \sin (\mu \pi a) A_{1}(a) d a$.
It is important to have an insight of the behavior of overall risk, at different environmental influence which infuse a stochastic nature in the proposed model. A mathematical analysis can be easily carried out to see the dynamical features by varying the periodicity of the stochastic process. As Eq. (10) indicates the behavior depends on the contribution of the weighing factor. The Figure 4 indicates that the maximum contribution of the weighing factor is obtained at minimum $\mu$ values. Together with the observation of sensitivity analysis of proportional constant, higher $\lambda$ values and lower $\mu$ values (i.e. $\mu \in \mathbb{Z}$ ), specifies that, the higher contribution of the accumulation at lower periodicity provides high risk of getting infected.

Figure 5 indicates the sensitivity of overall risk at different environmental influences controlled by varying periodicity. Again, it is readily observable that peaks slightly move towards higher ages with low periodicity.

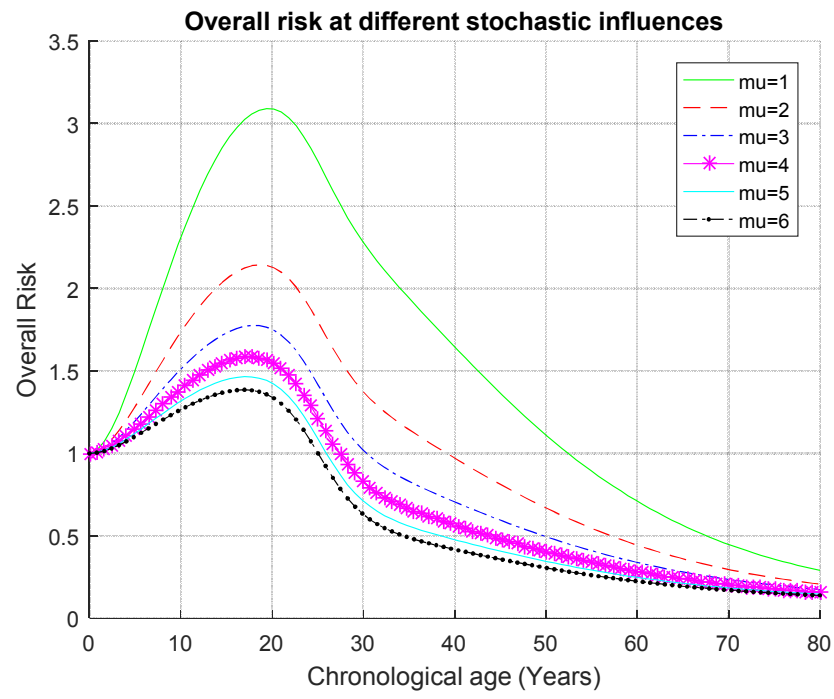

Figure 5. Sensitivity analysis on age-dependent risk of infectiousness at $\lambda=10$ and varying environmental influence | risk lessen from the acquired immunity when $\rho=0.4, \alpha=25$ and risk due to mobility when $\beta=11.5$ with $B_{1}(t)=\sin (\mu \pi t) ; \mu \in \mathbb{Z}$

\section{Discussion}

When the parsimonious modeling perspective is concerned, the proposed integral equation approach has a reasonable ability of incorporating biological, behavioral and environmental influences on age-dependent infectiousness of a disease. The model accuracy is a matter of choosing suitable curves for each influencing function.

This model can be used as a guide to formulate hypotheses and data collection strategies to measure the risk of a disease. For instance, when different immunity mechanisms play in different ages, $f(a)$ curve can be tested with different options. One motivation towards this is the fact that some diseases accountable with innate immunity in early ages and subsequently with acquired immunity. Different options on mobility related influences can be brought via $A_{k}$ functions. The degenerated kernel facilitates any finite number of such functions as it is easily solvable through a system of linear 
equations which provides a unique solution if the inequality $|I-\lambda A| \neq 0$ holds. Here $A=\left[A_{m k}\right]_{n \times n}$ where $A_{m k}=$ $\int_{0}^{L} A_{k}(t) B_{m}(t) d t$

The environmental influence which is not involved with age may vary due to the context addressed. Therefore, in this parsimonious modelling also there is a possibility of choosing a suitable stochasticity simply by averaging the range of randomness.

Here in this model, the proportional constant $\lambda$ is the only visible parameter to be estimated as all the other evaluations can be incorporated with functions proposed for any influential factor. As depicted in sensitivity analysis, modelbased experiments can be carried out to estimate $\lambda$ according to a selected set of influential functions with available prevalence data. Next, reliable predictions can be made using those models.

\section{Conclusion}

In this parsimonious approach, it is observed that the integral equations with degenerated kernel has a potential in modelling the behavior of infectious disease transmission. This approach shows the ability of collectively considering different factors relevant to age. The sensitivity analysis for $\lambda$ and $\mu$, indicates the behavior of the overall risk. It is observed that the risk may increase and saturation may vanish with higher $\lambda$ and $\mu$ values. Further the peaks slightly move towards higher ages with higher $\lambda$ and $\mu$ values. This illustrates that the possibility of identifying the required biological influence in order to not to get infected even with the maximum behavioral influence and different periodicity in environmental influences.

\section{References}

[1] N. Grassly, and C. Fraser, "Mathematical models of infectious disease transmission," Nature Reviews Microbiology, vol. 6, no. 6 , pp. 477-487, 2008.

[2] Dodd, P., Looker, C., Plumb, I., Bond, V., Schaap, A., Shanaube, K., Muyoyeta, M., Vynnycky, E., Godfrey-Faussett, P., Corbett, E., Beyers, N., Ayles, H. and White, R. (2015). Age- and Sex-Specific Social Contact Patterns and Incidence of Mycobacterium tuberculosis Infection. American Journal of Epidemiology, p. kwv160.
[3] Read, J., Lessler, J., Riley, S., Wang, S., Tan, L., Kwok, K., Guan, Y., Jiang, C. and Cummings, D. (2014). Social mixing patterns in rural and urban areas of southern China. Proceedings of the Royal Society B: Biological Sciences, 281 (1785), pp. 20140268-20140268.

[4] Pitzer, V. and Lipsitch, M. (2009). Exploring the relationship between incidence and the average age of infection during seasonal epidemics. Journal of Theoretical Biology, 260 (2), pp. 175-185.

[5] Chan, E. Michael, S. Pani, R. Norman, D. Bundy, P. Vanamail, K. Ramaiah, P. Das, and A. Srividya, "Epifil: a dynamic model of infection and disease in lymphatic filariasis," The American Journal of Tropical Medicine and Hygiene, vol. 59, no. 4, pp. 606-614, 1998.

[6] M. Gambhir, and E. Michael, "Complex Ecological Dynamics and Eradicability of the Vector Borne Macroparasitic Disease," Lymphatic Filariasis. PLoS ONE, vol. 3, no. 8, pp. 100-110, 2008.

[7] E. Shim, Z. Feng, M. Martcheva and C. Castillo-Chavez, "An age-structured epidemic model of rotavirus with vaccination," Journal of Mathematical Biology, vol. 53, no. 4, pp. 719-746, 2006.

[8] R. Norman, M. Chan, A. Srividya, S. Pani, K. Ramaiah, P. Vanamail, E. Michael, P. Das and D. Bundy, "EPIFIL: The development of an age-structured model for describing the transmission dynamics and control of lymphatic filariasis," Epidemiology and Infection, vol. 124, no. 3, pp. 529-541, 2000.

[9] J. Murray, An Introduction to Mathematical Biology. New York: Springer, 2001.

[10] Matt Keeling and Pej Rohani, Modelling Infectious Diseases. New Jersey: Princeton University Press, 2008.

[11] M. Li and X. Liu, "An SIR Epidemic Model with Time Delay and General Nonlinear Incidence Rate," Abstract and Applied Analysis, pp. 1-7, 2014.

[12] A. Jerri, Introduction to integral equations with applications. New York: Dekker, 1985.

[13] W. A. Stolk, "Lymphatic Filariasis: Transmission, Treatment and Elimination." (Ph.D. Thesis), Erasmus University Rotterdam, Netherlands, 2005. 